\title{
Characterization of the blue emission of Tm/Er co-implanted GaN
}

\author{
I.S. Roqan ${ }^{1}$, C. Trager-Cowan ${ }^{1}$, B. Hourahine ${ }^{1}$, K. Lorenz ${ }^{2}$, E. Nogales ${ }^{1}$, K.P. O’Donnell ${ }^{1}$, \\ R.W. Martin ${ }^{1}$, E. Alves ${ }^{2}$, S. Ruffenach ${ }^{3}$, O. Briot ${ }^{3}$. \\ ${ }^{1}$ Dept. of Physics, University of Strathclyde, Glasgow G4 0NG, UK. \\ ${ }^{2}$ ITN, Estrada Nacional 10, 2686-953 Sacavém, Portugal. \\ ${ }^{3}$ Groupe d'Etude des Semiconducteurs, Université Montpellier II, 34095 Montpellier, France.
}

\begin{abstract}
Comparative studies have been carried out on the cathodoluminescence (CL) and photoluminescence (PL) properties of $\mathrm{GaN}$ implanted with Tm and $\mathrm{GaN}$ co-implanted with $\mathrm{Tm}$ and a low concentration of Er. Room temperature CL spectra were acquired in an electron probe microanalyser to investigate the rare earth emission. The room temperature CL intensity exhibits a strong dependence on the annealing temperature of the implanted samples. The results of CL temperature dependence are reported for blue emission $(\sim 477 \mathrm{~nm})$ which is due to intra $4 f$-shell electron transitions $\left({ }^{1} \mathrm{G}_{4} \rightarrow{ }^{3} \mathrm{H}_{6}\right)$ associated with $\mathrm{Tm}^{3+}$ ions. The $477 \mathrm{~nm}$ blue CL emission is enhanced strongly as the annealing temperature increases up to $1200^{\circ} \mathrm{C}$. Blue PL emission has also been observed from the sample annealed at $1200^{\circ} \mathrm{C}$. To our knowledge, this is the first observation of blue PL emission from Tm implanted GaN samples. Intra- 4 f transitions from the ${ }^{1} D_{2}$ level ( $\sim 465 \mathrm{~nm}$ emission lines) of $\mathrm{Tm}^{3+}$ ions in GaN have been observed in GaN:Tm films at temperatures between $20-200 \mathrm{~K}$. We will discuss the temperature dependent $\mathrm{Tm}^{3+}$ emission in both GaN:Tm,Er and GaN:Tm samples.
\end{abstract}

\section{INTRODUCTION}

Extensive studies of GaN doped with rare earths (REs) (in-situ doped during growth or implanted), have produced red, green and blue (RGB) emission from $\mathrm{GaN}: \mathrm{Eu}, \mathrm{GaN}$ : Er and GaN:Tm, respectively. The aim of these studies is to produce multiple color capability based on $\mathrm{GaN}: \mathrm{RE}$, for example, fabrication of white light LEDs through the light emission from co-doped $\mathrm{GaN}: \mathrm{Eu}, \mathrm{Er}, \mathrm{Tm}$. For example, Steckl et al (2001), have reported on multiple color capability based on RE-doped GaN electroluminescence [1]. Intermediate colors from GaN:RE have been reported through MBE co-doping during growth, such as turquoise from $\mathrm{GaN}$ :Er,Tm and orange from GaN:Eu,Er [2]. The biggest challenge in the field of light emitting RE doped $\mathrm{GaN}$ is to obtain blue emission from GaN:Tm with high device efficiency. In this study, we present the RE luminescence properties of GaN co-implanted with Tm and a low concentration of Er.

REs in GaN replace gallium on a substitutional site of symmetry $\mathrm{C}_{3 v}$ [3]. The $4 f$ orbitals of $\mathrm{RE}$ ions incorporated into semiconductors are so strongly screened that energy levels of the $4 f$ configuration are only slightly perturbed compared to free-ion energy levels [3]. Therefore, the effect of the surrounding host is weak, yielding sharp luminescence lines. We are particularly interested in the persistence of such luminescence at elevated temperature, as required for device operation. Hence we will present here CL temperature-quenching studies. We have carried out preliminary studies of the PL and CL emission and thermal quenching of $\mathrm{Tm}^{3+}$ in the presence of $\mathrm{Er}^{3+}$, to investigate the effect of the presence of $\mathrm{Er}^{3+}$ ions on $\mathrm{Tm}^{3+}$ emission. 


\section{SAMPLES AND EXPERIMENTAL DETAILS}

Tm and Er were co-implanted into undoped AlN-capped GaN epilayers which were grown by metal organic chemical vapour deposition (MOCVD) on $c$-plane sapphire. The GaN and AlN cap are $2 \mu \mathrm{m}$ and a few tens of nanometer thick, respectively [4]. Tm ions were implanted using a $300 \mathrm{keV}$ ion beam. The SRIM simulations [5] predict that the ions stop at a depth of $54 \mathrm{~nm}$ underneath the AlN cap and $51 \mathrm{~nm}$ for uncapped samples. Whereas Rutherford Backscattering (RBS) depth-profiling shows the maximum ion concentration for uncapped material at a depth of $\sim 70 \mathrm{~nm}$ and $\sim 80 \mathrm{~nm}$ for capped materials, which is attributed to channeling effects [4]. The SRIM simulations and RBS show that Tm ions penetrate the AIN cap to stop in the GaN layer. Afterwards, samples were annealed for $20 \mathrm{~min}$. at $1200^{\circ} \mathrm{C}$ (sample A), $1100^{\circ} \mathrm{C}$ (sample B), and $1000^{\circ} \mathrm{C}$ (sample C), at 3.8 bar pressure of $\mathrm{N}_{2}$ gas. The samples were accidentally co-doped with low fluence of $\operatorname{Er}\left(<<1 \times 10^{15} \mathrm{at} / \mathrm{cm}^{2}\right)$. Reference sample D is Tm implanted into a $2 \mu \mathrm{m}$ undoped MOCVD GaN epilayer grown on $c$-plane sapphire. No capping layer was present for this sample. The implantation fluence was $3 \times 10^{15} \mathrm{at} / \mathrm{cm}^{2}$. The sample was annealed under the same conditions as sample $\mathrm{C}$.

CL measurements were carried out at room temperature, in a Cameca SX100 electron probe microanalyser. The spectra were acquired over a wavelength range of $430-830 \mathrm{~nm}$ with a spectral resolution of $1.2 \mathrm{~nm}$. CL temperature dependent measurements were carried out in a home built CL spectroscopy system. The spectra were acquired over a wavelength range of 460 $490 \mathrm{~nm}$ with a spectral resolution of $0.05 \mathrm{~nm}$, in the temperature range 20-300K. PL was excited using the $325 \mathrm{~nm}$ line of a He-Cd laser. PL spectra were acquired over a wavelength range of $460-490 \mathrm{~nm}$ in the temperature range $18-300 \mathrm{~K}$ with a spectral resolution of $0.4 \mathrm{~nm}$.

\section{EXPERIMENTAL RESULTS AND DISCUSSION}

The CL spectra of GaN:Tm,Er and GaN:Tm recorded at room temperature (RT) are shown in figures (1) and (2), respectively. CL spectra of Tm/Er co-implanted GaN at different annealing temperatures include two major lines of the $\mathrm{Tm}^{3+}$ ion, in the blue at $477 \mathrm{~nm}$ and near infrared (NIR) at $805 \mathrm{~nm}[6]$. The green lines $(537$ and $558 \mathrm{~nm})$ are related to intra $-4 f$ inner shell transitions of $\mathrm{Er}^{3+}$ ions [6].

As shown in figure (1) the $\mathrm{RE}^{3+}$ emissions are enhanced as the annealing temperature increases. A strong increase of CL intensity with annealing temperatures above $1000^{\circ} \mathrm{C}$ has also been reported for Eu implanted GaN [4].

Our assignment for the $\mathrm{RE}^{3+}$ transitions is based on comparison with data from the literature [6-10]. The blue line at $477 \mathrm{~nm}$ and the near infrared line at $805 \mathrm{~nm}$ shown in figure (1) are attributed to the intra- $4 f$ transitions $\left({ }^{1} \mathrm{G}_{4} \rightarrow{ }^{3} \mathrm{H}_{6}\right)$ and $\left({ }^{3} \mathrm{H}_{4} \rightarrow{ }^{3} \mathrm{H}_{6}\right)$ of $\mathrm{Tm}^{3+}$ ions, respectively [6]. The two green lines at $537 \mathrm{~nm}$ and $558 \mathrm{~nm}$ are attributed to the intra- $4 f$ transitions $\left({ }^{2} \mathrm{H}_{11 / 2} \rightarrow{ }^{4} \mathrm{I}_{15 / 2}\right)$ and $\left({ }^{4} \mathrm{~S}_{3 / 2} \rightarrow{ }^{4} \mathrm{I}_{15 / 2}\right)$ of $\mathrm{Er}^{3+}$ ions, respectively [6]. The line at $694 \mathrm{~nm}$, which appears in all spectra, is the $\mathrm{Cr}^{3+}$ emission line originating from the sapphire substrate of the samples [6].

Figure (2) shows the CL spectrum of GaN implanted with a fluence $3 \times 10^{15} \mathrm{Tm}$ at $/ \mathrm{cm}^{2}$ with a subsequent $1000^{\circ} \mathrm{C}$ anneal temperature (sample D). Comparing the spectra of $\mathrm{Tm}$ implanted with that of Tm/Er co-implanted GaN, we do not observe any significant differences in the $\mathrm{Tm}^{3+}$ emission lines. Comparing the spectra of Tm/Er implanted GaN (sample C) and Tm implanted GaN (sample D) shown in figures (1 and 2), respectively, reveals that the ratio of the 
$477 \mathrm{~nm}$ to $805 \mathrm{~nm}$ peak intensities in GaN:Tm is $\sim 0.4$, whereas in GaN:Tm/Er (sample C) it is 0.6. This ratio increases in the GaN:Tm,Er samples from $\sim 0.6$ (sample C) to $\sim 1$ (sample A) as the anneal temperature increases from 1000 to $1200^{\circ} \mathrm{C}$, as shown in figure (1). Lorenz, et al, observed a similar dependence on implantation temperature in Tm implanted GaN films [11]. For the sample annealed at $1200^{\circ} \mathrm{C}$, a broad emission band emerges in the wavelength range $500-650 \mathrm{~nm}$.

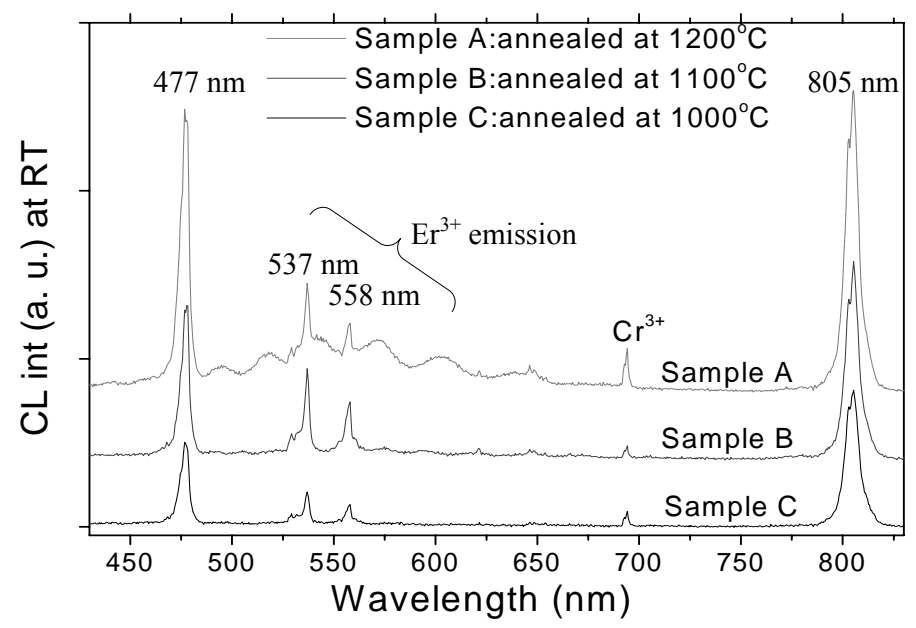

Figure 1. Room temperature CL spectra of Tm/Er implanted $\mathrm{GaN}$ at different anneal temperature, showing $\mathrm{Tm}^{3+}$ blue and NIR emissions at 477 and $805 \mathrm{~nm}$ and $\mathrm{Er}^{3+}$ green emission at 537 and $558 \mathrm{~nm}$. The line marked $\mathrm{Cr}^{3+}$ originates in the sapphire substrate.

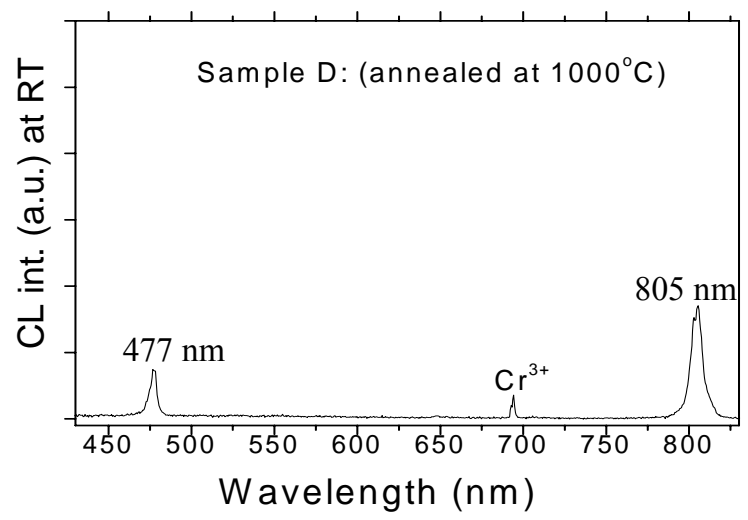

Figure 2. Room temperature CL spectra of Tm implanted GaN, showing $\mathrm{Tm}^{3+}$ blue and NIR emissions at 477 and $805 \mathrm{~nm}$.

Figure (3a) shows high resolution CL spectra of the blue $\mathrm{Tm}^{3+}$ emission of sample A as a function of temperature in the range $20-300 \mathrm{~K}$. The $\mathrm{Tm}^{3+} \mathrm{CL}$ spectra exhibit a number of different narrow lines in the blue region, particularly at low temperature. The different emission lines could possibly originate from different $\mathrm{Tm}^{3+}$ centres formed in $\mathrm{GaN}$ or be due to crystal field splitting of the $\mathrm{Tm}^{3+}$ levels [12-14].

The group of lines at 479.6 and $479.2,478$ and $476 \mathrm{~nm}$ is assigned to ${ }^{1} \mathrm{G}_{4} \rightarrow{ }^{3} \mathrm{H}_{6}$ transitions of $\mathrm{Tm}^{3+}$ [6-8]. The shorter wavelength group of lines (from 462 to $466.7 \mathrm{~nm}$ ) can be attributed to ${ }^{1} \mathrm{D}_{2} \rightarrow{ }^{3} \mathrm{~F}_{4}$ transitions of $\mathrm{Tm}^{3+}[7,8,12]$. As can be seen from the energy diagram, 
shown in figure (4), the ${ }^{1} \mathrm{D}_{2}$ state is very close in energy to the band gap of GaN. The ${ }^{1} \mathrm{D}_{2} \rightarrow{ }^{3} \mathrm{~F}_{4}$ transitions were not identified in GaN:Tm films in previous studies [6-10], although a strong emission has been observed due to transitions related to the ${ }^{1} \mathrm{D}_{2}$ level in GaN:Tm quantum dots [8]. However, the final identification of these transitions requires further investigation.

Different peaks have different thermal quenching behavior as indicating in Figures. (3a) and ( $3 b$ ). The line due to the ${ }^{1} \mathrm{D}_{2} \rightarrow{ }^{3} \mathrm{~F}_{4}$ transitions is completely quenched and vanishes at temperatures above $200 \mathrm{~K}$ as shown in figure (3a). This may be due to the fact that the ${ }^{1} \mathrm{D}_{2}$ level is close to the band gap energy of GaN. Some of the lines related to the ${ }^{1} \mathrm{G}_{4}$ level exhibit intermediate behavior as shown in figure (3b). The emission line at $479.6 \mathrm{~nm}$ due to the transition from ${ }^{1} \mathrm{G}_{4}$ decreases in intensity as the temperature increases, whereas the intensity of the peak at $478 \mathrm{~nm}$, which is related to the ${ }^{1} \mathrm{G}_{4} \rightarrow{ }^{3} \mathrm{H}_{6}$ transitions, grows continuously to reach its maximum at $100 \mathrm{~K}$. For temperatures up to $300 \mathrm{~K}$, the decline of the $478 \mathrm{~nm}$ line is quite slow: at room temperature it is about $60 \%$ of its maximum at $100 \mathrm{~K}$.

A new line peaking at $476 \mathrm{~nm}$ related to a ${ }^{1} \mathrm{G}_{4}$ transition emerges when the temperature reaches $40 \mathrm{~K}$ and grows slowly up to a temperature of $100 \mathrm{~K}$. It saturates as the temperature increases up to $300 \mathrm{~K}$. The nature of this line has yet to be determined. It may be related to thermal population of higher crystalline Stark split levels of ${ }^{1} \mathrm{G}_{4}$, or $\mathrm{Tm}^{3+}$ centres of different symmetry.

The emission lines at 478 and $476 \mathrm{~nm}$, due to ${ }^{1} \mathrm{G}_{4} \rightarrow{ }^{3} \mathrm{H}_{6}$ transitions, show remarkably small thermal quenching when compared to the emission of the $479.6 \mathrm{~nm}$ line (figure (3b)). The thermal quenching mechanisms are proposed to be mostly nonradiative recombination of the excited states of a localized $\mathrm{RE}^{3+}$ center in GaN [13]. These mechanisms could comprise multiphonon relaxation processes, and a migration of energy and cross-relaxation processes. If the energy gap to the next lower state is sufficiently large, the nonradiative multiphonon transition rate is negligible compared to the radiative rate [13]. This explains the weak temperature quenching of transitions of ${ }^{1} \mathrm{G}_{4}$ level and its strong CL emission at RT as shown in figure (1).
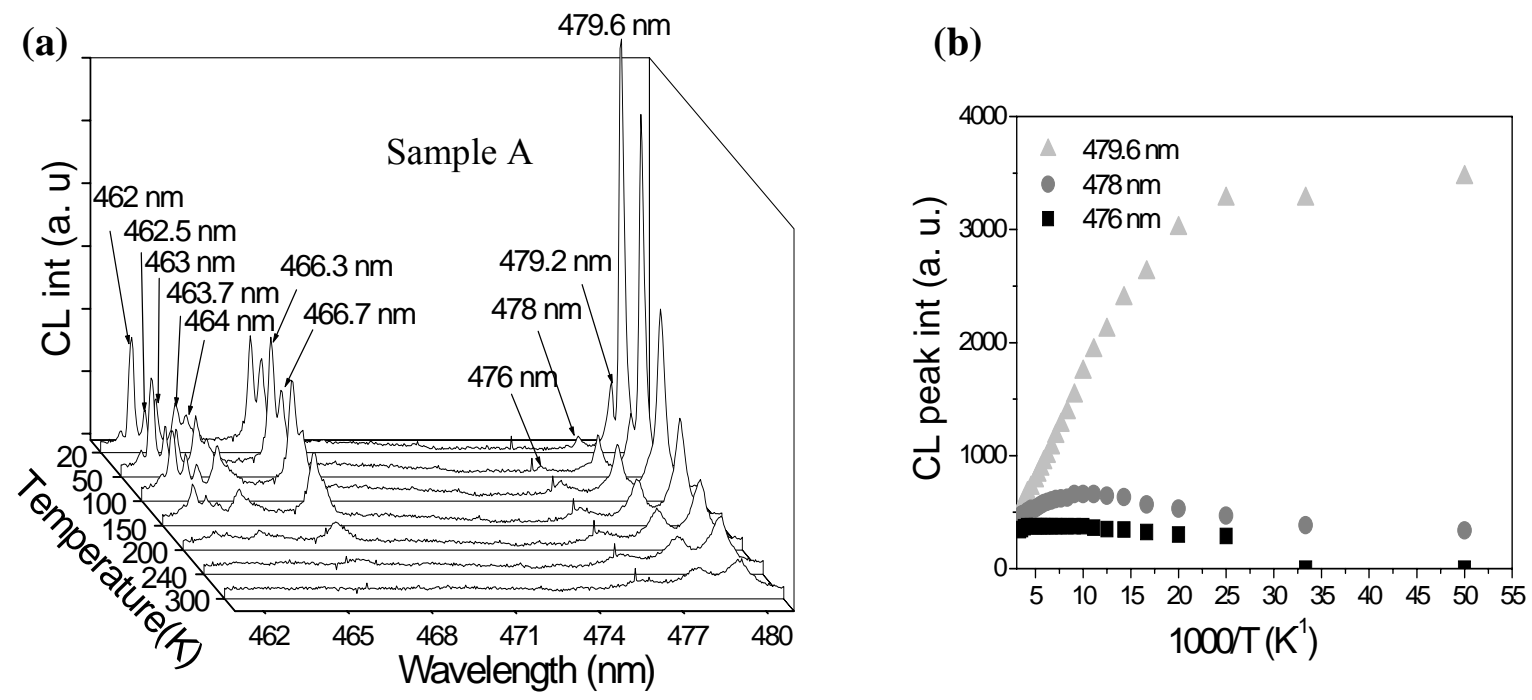

Figure 3. (a) Temperature dependence of CL spectra of GaN implanted with Tm and Er (annealed at $1200^{\circ} \mathrm{C}$ ). (b) The temperature dependence of CL peaks intensities related to ${ }^{1} \mathrm{G}_{4} \rightarrow{ }^{3} \mathrm{H}_{6}$ transitions. 


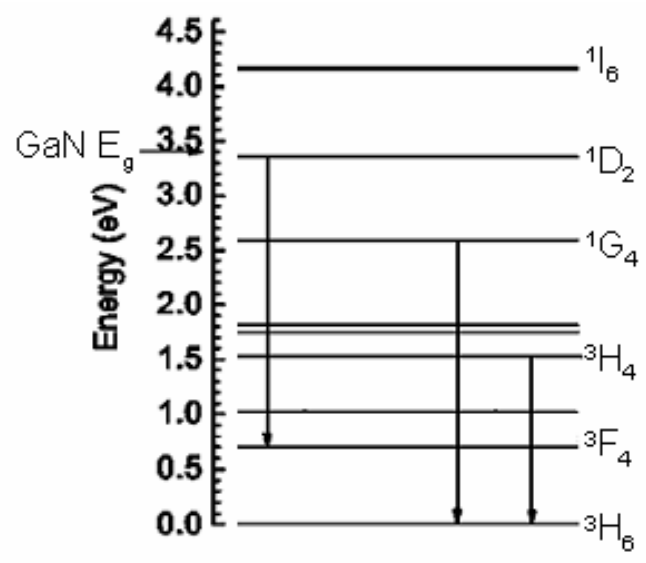

Figure 4. Free ion energy levels diagram of $\mathrm{Tm}^{3+}$ ions and assigned transitions of Tm implanted GaN. The band gap of $\mathrm{GaN}$ is also indicated in the figure, relative to the ${ }^{3} \mathrm{H}_{6}$ ground state of the free ion.

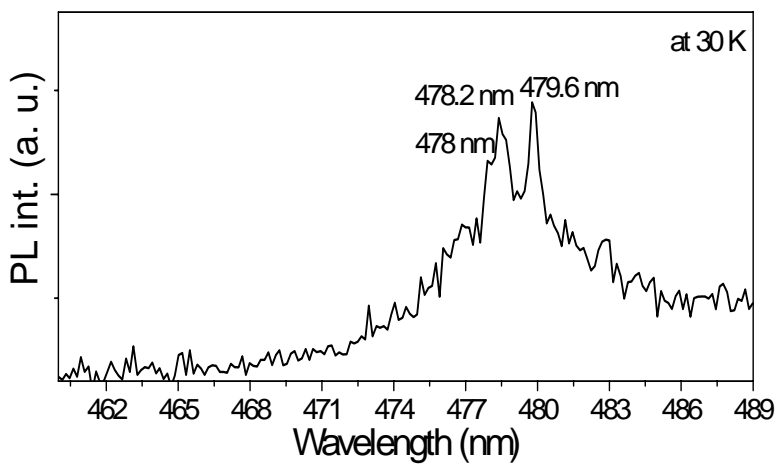

Figure 5. PL spectrum of $\mathrm{Tm} / \mathrm{Er}$ implanted $\mathrm{GaN}$ and annealed at $1200^{\circ} \mathrm{C}$ in blue region. $\mathrm{Tm}^{3+}$ emission due to ${ }^{1} \mathrm{G}_{4} \rightarrow{ }^{3} \mathrm{H}_{6}$ transitions is observed in PL. The emission due to ${ }^{1} \mathrm{D}_{2} \rightarrow{ }^{3} \mathrm{~F}_{4}$ transitions has been not observed.

Figure (5) shows the PL spectrum of $\mathrm{Tm}^{3+}$ ions in GaN:Tm,Er (sample A) at low temperature. A weak luminescence was observed related to ${ }^{1} \mathrm{G}_{4} \rightarrow{ }^{3} \mathrm{H}_{6}$ transitions. This is the first observation of PL blue emission due to ${ }^{1} \mathrm{G}_{4} \rightarrow{ }^{3} \mathrm{H}_{6}$ transitions from Tm implanted GaN to our knowledge. The transitions originating on the ${ }^{1} \mathrm{D}_{2}$ level are not seen. This illustrates the complexity of the energy transfer when comparing PL and CL. Future study of selective excitation and time-resolved temperature dependence should clarify our tentative assignments and the energy transfer process.

To investigate the effect of the presence of $\mathrm{Er}^{3+}$ ions on $\mathrm{Tm}^{3+}$ emission, we studied the thermal quenching of Tm (only) implanted GaN which has the same implantation and annealing conditions as (sample C) GaN:Tm,Er. From the CL spectra studies of $\mathrm{Tm}^{3+}$ emission in GaN:Tm in the temperature range $20-300 \mathrm{~K}$, we observed the same peaks in the blue region in CL at low temperature. These lines have the same thermal quenching behavior observed in GaN:Tm,Er as shown in figures (3a) and (3b). Therefore, a low concentration of $\mathrm{Er}^{3+}$ does not notably influence the thermal quenching of $\mathrm{Tm}^{3+}$ emission. 


\section{CONCLUSIONS}

We have carried out preliminary studies of the PL and CL emission and thermal quenching of $\mathrm{Tm}^{3+}$ in the presence of a low concentration of $\mathrm{Er}^{3+}$ ions. We found that the ratio of $\mathrm{Tm}^{3+}$ blue emission to NIR increased as the anneal temperature increased. From our studies we show that the energy transfer to ${ }^{1} \mathrm{D}_{2}$ level in blue region of $\mathrm{Tm}^{3+}$ ion is allowed in GaN:Tm,Er and GaN:Tm films. Blue PL Tm ${ }^{3+}$ emission has been observed from implanted GaN films for first time to our knowledge. We compared the luminescence properties of $\mathrm{Tm} / \mathrm{Er}$ co-implanted $\mathrm{GaN}$ with Tm implanted GaN to investigate the effect of the presence of $\mathrm{Er}^{3+}$ ions on $\mathrm{Tm}^{3+}$ emission. We found that co-implanted with low concentration Er did not have a detrimental effect on the Tm luminescence temperature dependence.

\section{REFERENCES}

[1] A. J. Steckl, J. Heikenfeld, D. S. Lee and M. Garter, Mat. Sci. Eng. B 81, 97 (2001).

[2] A. J. Steckl and J. M. Zavada, MRS Bull. 24, 33 (1999).

[3] H. J. Lozykowski W. M. Jadwisienczak and I Brown, J. App. Phys. 88, 210 (2000).

[4] K Lorenz, U. Wahl, E. Alves, S. Dalmasso, R. W. Martin, K. P. O'Donnell, S. Ruffenach and O. Briot. Appl. Phys. Lett. 85, 2712 (2004).

[5] J. F. Ziegler, J. P. Biersack, U. Littmark, The stopping and range of ions in solids (Pergamon Press, New York, 1985).

[6] H. J. Lozykowski W. M. Jadwisienczak and I Brown, Appl. Phys. Lett. 74, 1129 (1999).

[7] U. Hommerich, Ei Ei Nyein, D. S. Lee, A. J. Steckl and J. M. Zavada, Appl. Phys. Lett. 83, 4556 (2003).

[8] T. Andreev, Y. Hori, X. Biquard, E. Monroy, D. Jalabert, A. Farchi, M. Tanaka, O. Oda, Le Si Dang, and B. Daudin, Phys. Rev. B 71, 115310 (2005).

[9] U. Hömmerich, Ei Ei Nyein, D. S. Lee, J. Heikenfeld, A. J. Steckl and J. M. Zavada, Mater. Sci. Eng. B 105, 91 (2003).

[10] D. S. Lee and A. J. Steckl, Appl. Phys. Lett. 832094 (2003).

[11] K. Lorenz, U. Wahl, E. Alves, S. Dalmasso, R.W. Martin, K.P. O`Donnell, MRS Symp. Proc. 798, Y5.4 (2004).

[12] M. D. Seltzer, J. B. Gruber and M. E. Hills, J. Appl. Phys. 74, 2821 (1993).

[13] H. J. Lozykowski, Phys. Rev. B 48, 17758 (1993).

[14] A.J. Kenyon, Progress in Quantum Electronics 26, 225 (2002). 